\title{
Performance of Block Diagonalization Broadcasting Scheme for Multiuser MIMO System Operating in Presence of Spatial Correlation and Mutual Coupling
}

\author{
Feng Wang, Marek E. Bialkowski, Xia Liu \\ School of Information Technology \& Electrical Enginnering, University of Queensland, Brisbane, Australia \\ Email: \{fwang,meb,xialiu\}@itee.uq.edu.au \\ Received December 31, 2009; revised January 30, 2010; accepted February 27, 2010
}

\begin{abstract}
In this paper, the capacity of a multiuser Multiple Input Multiple Output (MIMO) system employing the block diagonalization broadcasting scheme in presence of spatial correlation and mutual coupling is investigated. It is shown by computer simulations that, in general, the presence of spatial correlation decreases the capacity of a multiuser MIMO system. However, for some particular antenna element spacing mutual coupling decreases the spatial correlation rendering an increased capacity. The optimized diagonalization broadcasting technique with a two-stage power allocation scheme is proposed and verified. The presented simulations results confirm the advantage of the proposed broadcasting scheme.
\end{abstract}

Keywords: Multiuser MIMO, Block Diagonalizaiton, Mutual Coupling, Dirty Paper Coding

\section{Introduction}

It has been shown via theoretical derivations as well as by experiment that using multiple element antennas with a suitable signal transmission scheme in a rich scattering propagation environment can enhance peer-to-peer communication without the use of extra frequency bandwidth $[1,2]$. This potential of multiple input multiple output (MIMO) communication systems can be used to advantage using two alternative approaches. In one approach, the signal transmission quality via diversity can be improved. Alternatively for a chosen quality factor such as bit error rate (BER), the data rate can be increased by a stream multiplexing transmission.

Most recent studies on MIMO focus on multiuser systems. For a multiuser MIMO system, allocation of the channel resources among independent users either in the form of multiple accesses (uplink) or broadcasting (downlink) is considered. The information theory hints that the broadcasting case is by far the most challenging. In this case, an inter-user interference occurs due to the spatially multiplexed transmitted signals at a base station (BS). For the Gaussian MIMO broadcasting channels, it has been proved that dirty paper coding (DPC) [3] can achieve the available capacity [4]. However, to deploy DPC in a real system is challenging due to the high complexity and computational burden on successive encod- ing and decoding. An alternative strategy is the block diagonalization (BD) $[5,6]$. Compared with DPC scheme, $\mathrm{BD}$ is a suboptimal technique with much reduced complexity. Using this technique, signals are transmitted only to desired users. In turn, null steering is applied to other users by decomposing the multiuser channel into a group of parallel single user MIMO channels. To achieve such decomposition, BS needs to select a suitable beamforming matrix for each user. The matrix is vertical to the space spanned by other users' channels matrices. If the channel matrices of all the scheduled users are perfectly known at the transmitter, the inter-user interference can be eliminated by $\mathrm{BD}$, rendering a simple receiver structure.

Because of its simplicity and good performance, BD is under constant research. In [7], the imperfect channel state information (CSI) assumption was used while investigating BD. The effect of outdated CSI at transmitter on multiuser MIMO system with BD was reported. In [8], a $\mathrm{BD}$ algorithm that accounts for the presence of othercell interferences was proposed under the assumption that the transmitter has full CSI and the information about the interference plus noise covariance matrix for in-cell users. Most of the research on BD for multiuser MIMO systems was done by neglecting interactions within the transmitting and receiving array antennas and between the array antennas and scatterers. When a 
MIMO transceiver has to be of compact size interactions within the transmitting or receiving array antennas have to be taken into account. The small inter-element spacing in the antenna array in such transceivers renders mutual coupling. The effect of mutual coupling on a pointto-point MIMO system has been investigated in many works, such as $[9,10,11]$. In this paper, a BD algorithm that accounts for the effect of spatial correlation and mutual coupling in array antennas is presented and its performance is evaluated with respect to the overall system capacity.

The paper is organized as follows. Section 2 describes a multiuser MIMO system model including the channel model with spatial correlation. Section 3 describes interactions between the array elements and scatterers in the propagation environment in which mutual coupling effects cannot be neglected. Section 4 gives details of the BD algorithm that accounts for the effect of mutual coupling. Section 5 quantifies the effect of mutual coupling by presenting numerical results. Section 6 summarizes the findings of the undertaken research.

\section{System Model}

\subsection{Signal Model}

A narrowband multiuser system is assumed. It is postulated that it is created around a base station (BS) with $L$ downlink mobile users. The base station includes $N$ transmitting antennas. At time $t, K$ mobile stations (MS) from $L$ available users are scheduled to be serviced by BS. The $k$-th mobile station (MS) employs $M_{\mathrm{k}}$ antennas. The transmitted signal intended for the $k$-th mobile station is denoted by a $Q_{\mathrm{k}} \times 1$ dimensional vector $\mathbf{x}_{k}$ which is weighted by an $N \times Q_{\mathrm{k}}$ pre-processing matrix $\mathbf{W}_{k}$ before transmission. $Q_{\mathrm{k}}$ is the number of parallel data symbols transmitted simultaneously to the $k$-th MS. The MIMO channel between the BS the $k$-th MS is described by the complex matrix $\mathbf{H}_{k}$, whose $(i, j)$ th entries represent the complex gain between the $j$-th transmit antenna at BS and $i$-th antenna at $k$-th MS. It is assumed that different MS experience independent fading. The received signal at $k$-th MS can be presented by

$$
\begin{aligned}
\mathbf{y}_{k} & =\mathbf{H}_{k} \sum_{k=1}^{K} \mathbf{W}_{k} \mathbf{E}_{k} \mathbf{x}_{k}+\mathbf{n}_{k} \\
& =\mathbf{H}_{k} \mathbf{W}_{k} \mathbf{E}_{k} \mathbf{x}_{k}+\sum_{j=1, j \neq k}^{K} \mathbf{H}_{k} \mathbf{W}_{j} \mathbf{E}_{j} \mathbf{x}_{j}+\mathbf{n}_{k}
\end{aligned}
$$

where trace $\left(\mathrm{E}_{\mathrm{k}} \mathrm{E}_{\mathrm{k}}^{\dagger}\right)=p_{k}$ is the power transmitted to the $k$ th MS. $\mathbf{n}_{k}$ is the additive Gaussian white noise (AWGN) vector, whose elements are independent identical distribution (i.i.d.) zero-mean circularly symmetric complex Gaussian random variables with variance $\boldsymbol{\sigma}_{\mathrm{n}}{ }^{2}$.

\subsection{Channel Model}

The channel matrix $\mathbf{H}_{k}$ describing the channel properties between BS and the $k$ th MS is influence by the transmitting and receiving antenna array configurations and a signal propagation environment. It is assumed that the $\mathrm{BS}$ and MSs are equipped with wire dipoles arranged in liner arrays. The length of each dipole element is assumed to be half wavelength. Also, the links between BS and different MSs do not share the same scattering environment. This assumption confirms the earlier assumed independent signal fading for different MSs. For each link, the Kronecker channel model $[5,12]$ is assumed. In this model, the correlations at transmitter and receiver sides are independent and the channel matrix $\mathbf{H}_{k}$ is represented as

$$
\mathbf{H}_{k}=\left(\mathbf{R}_{M S}^{k}\right)^{1 / 2} \mathbf{G}_{\mathbf{H}} \mathbf{R}_{B S}^{1 / 2}
$$

where $\mathbf{G}_{\mathbf{H}}$ is a matrix with i.i.d. Gaussian entries with zero mean and unit variance and $\mathbf{R}_{M S}^{k}$ and $\mathbf{R}_{B S}$ are spatial correlation matrices at the $k$ th $\mathrm{MS}$ and $\mathrm{BS}$, respectively. In a rich scattering environment, the correlation for any pair of dipole element with spacing $d_{m, n}$ can be obtained using Clark's model and are given by a Bessel function

$$
\rho_{m, n}=J_{0}\left(k d_{m, n}\right)
$$

Using (3), the correlation matrix for the $k$ th MS can be generated as

$$
\mathbf{R}_{M S}^{k}=\left[\begin{array}{ccc}
\rho_{1,1} & \cdots & \rho_{1, M_{k}} \\
\vdots & \ddots & \vdots \\
\rho_{M_{k}, 1} & \cdots & \rho_{M_{k}, M_{k}}
\end{array}\right]
$$

In turn, the correlation matrix for BS can be obtained from

$$
\mathbf{R}_{B S}=\left[\begin{array}{ccc}
\rho_{1,1} & \cdots & \rho_{1, N} \\
\vdots & \ddots & \vdots \\
\rho_{N, 1} & \cdots & \rho_{N, N}
\end{array}\right]
$$

\section{Mutual Coupling}

For the array formed by linear parallel wire dipoles, the mutual coupling matrix can be expressed using electromagnetic and circular theory described in [9]

$$
\boldsymbol{C}=\left(\mathrm{Z}_{A}+\mathrm{Z}_{T}\right)\left(\mathbf{Z}+\mathrm{Z}_{T} \boldsymbol{I}_{M}\right)^{-1}
$$

where $Z_{A}=73+j 42.5[\Omega]$ is the element impedance in isolation and $Z_{T}$ is impedance of the receiver at each element. It is chosen to be the complex conjugate of $Z_{A}$ to obtain the impedance match. $\mathbf{Z}$ is the mutual impedance 
matrix with all the diagonal elements equal to $Z_{A}+Z_{T}$, its non-diagonal elements $Z_{n m}$ are decided by the physical parameters including dipole length, the horizontal distance between the two dipoles. For a side-by-side array configuration and dipole length $l$ equals to $0.5 \lambda, Z_{n m}$ is given by $[9,10]$

$$
Z_{m n}= \begin{cases}30\left[0.5722+\ln (2 \beta l)-C_{i}(2 \beta l)\right] & \\ +j\left[30 S_{i}(2 \beta l)\right], & m=n \\ 30\left[2 C_{i}\left(u_{0}\right)+C_{i}\left(u_{1}\right)-C_{i}\left(u_{2}\right)\right] & \\ +j\left[302 S_{i}\left(u_{0}\right)-S_{i}\left(u_{1}\right)-S_{i}\left(u_{2}\right)\right] & \end{cases}
$$

where $\beta=2 \pi \lambda$ is the wave number and $C_{i}(u)$ and $S_{i}(u)$ are the cosine and sine integral, respectively, given as

$$
\begin{aligned}
& C_{i}(u)=\int_{\infty}^{u} \frac{\cos (x)}{x} d x \\
& S_{i}(u)=\int_{0}^{u} \frac{\sin (x)}{x} d x
\end{aligned}
$$

and the constants are given by [10]

$$
\begin{aligned}
& u_{0}=\beta d_{h} \\
& u_{1}=\beta\left(\sqrt{d_{h}^{2}+l^{2}}+l\right) \\
& u_{2}=\beta\left(\sqrt{d_{h}^{2}+l^{2}}-l\right)
\end{aligned}
$$

where $d_{h}$ is the horizontal distance between the two dipole antennas.

\section{Block Diagonalizaiton with Mutual Coupling}

We assume at time $t, K$ mobile stations (MS) from $L$ available users are scheduled to be serviced by BS. To ensure the sufficient freedom for BS to perform BD over the $K$ scheduled MSs, it is assumed that

$$
\sum_{k=1}^{K} M_{k} \leq N
$$

With spatial correlation and mutual coupling taken into account, the received signal at $k$ th MS described by (1) can be rewritten as

$$
\begin{aligned}
\mathbf{y}_{k}= & \mathbf{C}_{M S}^{k}\left(\mathbf{R}_{M S}^{k}\right)^{1 / 2} \mathbf{G}_{\mathbf{H}} \mathbf{R}_{B S}^{1 / 2} \mathbf{C}_{B S} \sum_{k=1}^{K} \mathbf{W}_{k} \mathbf{E}_{k} \mathbf{x}_{k}+\mathbf{n}_{k} \\
= & \mathbf{C}_{M S}^{k}\left(\mathbf{R}_{M S}^{k}\right)^{1 / 2} \mathbf{G}_{\mathbf{H}} \mathbf{R}_{B S}^{1 / 2} \mathbf{C}_{B S} \mathbf{W}_{k} \mathbf{E}_{k} \mathbf{x}_{k} \\
& +\mathbf{C}_{M S}^{k}\left(\mathbf{R}_{M S}^{k}\right)^{1 / 2} \mathbf{G}_{\mathbf{H}} \mathbf{R}_{B S}^{1 / 2} \mathbf{C}_{B S} \sum_{j=1, j \neq k}^{K} \mathbf{W}_{j} \mathbf{E}_{j} \mathbf{x}_{j}+\mathbf{n}_{k}
\end{aligned}
$$

where, $\mathbf{C}_{B S}$ and $\mathbf{C}_{M S}^{k}$ are the mutual coupling matrices for the dipole element array at BS and the $k$ th $\mathrm{MS}$, respectively. $\mathbf{W}_{k}$ is the beamforming matrix at BS for the $k$ th MS. To eliminate the interference from the signals transmitted to other MSs, the key idea in the block diagonalization is to zero-force the interference by imposing the following condition

$$
\mathbf{C}_{M S}^{k}\left(\mathbf{R}_{M S}^{k}\right)^{1 / 2} \mathbf{G}_{\mathbf{H}} \mathbf{R}_{B S}^{1 / 2} \mathbf{C}_{B S} \mathbf{W}_{j}=\mathbf{0}(j \neq k, 1 \leq k, j \leq K)
$$

when the mutual coupling and correlation is taken into account,

$$
\begin{aligned}
& \overline{\mathbf{H}}_{k}=\mathbf{C}_{M S}^{k}\left(\mathbf{R}_{M S}^{k}\right)^{1 / 2} \mathbf{G}_{\mathbf{H}} \mathbf{R}_{B S}^{1 / 2} \mathbf{C}_{B S} \\
& \tilde{\mathbf{H}}=\left[\overline{\mathbf{H}}_{1}, \overline{\mathbf{H}}_{2}, \cdots, \overline{\mathbf{H}}_{K}\right]^{T} \\
& \mathbf{W}=\left[\mathbf{W}_{1}, \mathbf{W}_{2}, \cdots, \mathbf{W}_{K}\right]
\end{aligned}
$$

where $(\bullet)^{\mathrm{T}}$ donates the matrix transpose operation. By including the condition given by (12), the effective channel matrix for the multiuser MIMO system with $K$ MSs can be represented by a $\sum \mathrm{M}_{k} \times \sum \mathrm{M}_{k}$ matrix, given as

$$
\begin{aligned}
\mathbf{D} & =\tilde{\mathbf{H}} \mathbf{W} \\
& =\left[\begin{array}{cccc}
\overline{\mathbf{H}}_{1} \mathbf{W}_{1} & \overline{\mathbf{H}}_{1} \mathbf{W}_{2} & \cdots & \overline{\mathbf{H}}_{1} \mathbf{W}_{K} \\
\overline{\mathbf{H}}_{2} \mathbf{W}_{1} & \overline{\mathbf{H}}_{2} \mathbf{W}_{2} & \cdots & \overline{\mathbf{H}}_{2} \mathbf{W}_{K} \\
\vdots & \vdots & \ddots & \vdots \\
\overline{\mathbf{H}}_{K} \mathbf{W}_{1} & \cdots & \cdots & \overline{\mathbf{H}}_{K} \mathbf{W}_{K}
\end{array}\right]
\end{aligned}
$$

By using (12), Equation (14) can be rewritten as

$$
\mathbf{D}=\left[\begin{array}{cccc}
\overline{\mathbf{H}}_{1} \mathbf{W}_{1} & \mathbf{0} & \mathbf{0} & \mathbf{0} \\
\mathbf{0} & \overline{\mathbf{H}}_{2} \mathbf{W}_{2} & \mathbf{0} & \mathbf{0} \\
\mathbf{0} & \mathbf{0} & \ddots & \mathbf{0} \\
\mathbf{0} & \mathbf{0} & \mathbf{0} & \overline{\mathbf{H}}_{K} \mathbf{W}_{K}
\end{array}\right]
$$

At this point it is important to comment whether the condition (12) can be met in practice. From the theory of antennas it is known that an $N$-element array antenna is capable of forming $N-1$ nulls. This means that in the strict sense, the BS having an $N$-element array is able only to null up to $N-1$ MSs. For a larger number of MSs, the condition (12) has to be compromised. In such a case, the BS can direct low sidelobes instead of nulls towards undesired users. In further considerations, it is assumed that the number of MSs served by BS is such that the condition (12) is met.

\subsection{Calculation of Beamforming Matrices}

In order to transmit a signal only to the desired MS while steering nulls to the remaining MSs, the beamforming matrix for the desired MS should be orthogonal to the space spanned by the channel matrices of the undesired MSs. We define the channel matrix as

$$
\tilde{\mathbf{H}}_{k}=\left[\overline{\mathbf{H}}_{1} \cdots \overline{\mathbf{H}}_{k-1}, \overline{\mathbf{H}}_{k+1}, \cdots, \overline{\mathbf{H}}_{K}\right]^{T}
$$


which is obtained by removing the channel matrix for the $k$ th MS from $\tilde{\mathbf{H}}$. Performing the eigenvalue decomposition (EVD) over the $N \times N$ non-negative Hermitian Matrix, one obtains

$$
\tilde{\mathbf{H}}_{k}^{\dagger} \tilde{\mathbf{H}}_{k}=\left[\tilde{\mathbf{V}}_{k} \mathbf{V}_{k}\right]\left[\begin{array}{cc}
\boldsymbol{\Sigma} & \mathbf{0} \\
\mathbf{0} & \mathbf{0}
\end{array}\right]\left[\begin{array}{c}
\tilde{\mathbf{V}}_{k}^{\dagger} \\
\mathbf{V}_{k}^{\dagger}
\end{array}\right]
$$

where $(\bullet)^{\dagger}$ denotes the conjugate transpose operation.

It can bee seen that $\mathbf{V}_{k}$ is a matrix with the dimension of $N \times M_{k}$. Its columns correspond to those zero eigenvalues. By letting $\mathbf{W}_{k}=\mathbf{V}_{k}$, a perfect null steering to all the undesired $K-1$ MSs can be achieved. By repeating the steps represented by Equation (15) and (16), all the $K$ beamforming matrices can be obtained. In this way, as shown in Equation (14), the multiuser MIMO downlink system is decomposed into $K$ independent single-user MIMO systems.

\subsection{Overall Capacity of Multiuser MIMO Broadcasting with Block Diagonalization}

For the case of a multiuser MIMO downlink system which is decomposed into $K$ independent single-user MIMO systems by block diagonalization, the overall capacity can be obtained as a sum of individual links capacities, as expressed by

$$
C_{\text {sum }}=\sum_{k=1}^{K} \log _{2} \operatorname{det}\left(\mathbf{I}+\frac{1}{\sigma_{n}^{2}} \mathbf{E}_{k}^{\dagger} \mathbf{W}_{k}^{\dagger} \mathbf{H}_{k}^{\dagger} \mathbf{R}_{\mathbf{x x}}^{k} \mathbf{H}_{k} \mathbf{W}_{k} \mathbf{E}_{k}\right)
$$

With mutual coupling and spatial correlations taken into account, (18) can be rewritten as

$$
C_{\text {sum }}=\sum_{k=1}^{K} \log _{2} \operatorname{det}\left(\mathbf{I}+\frac{1}{\sigma_{n}^{2}} \mathbf{E}_{k}^{\dagger} \mathbf{W}_{k}^{\dagger} \overline{\mathbf{H}}_{k}^{\dagger} \mathbf{R}_{\mathbf{x x}}^{k} \overline{\mathbf{H}}_{k} \mathbf{W}_{k} \mathbf{E}_{k}\right)
$$

where $\mathbf{R}_{\mathbf{x x}}^{k}$ is the $k$ th MS's input covariance matrix. The capacity for $k$ th MS is

$$
C_{k}=\log _{2} \operatorname{det}\left(\mathbf{I}+\frac{1}{\sigma_{n}^{2}} \mathbf{E}_{k}^{\dagger} \mathbf{W}_{k}^{\dagger} \overline{\mathbf{H}}_{k}^{\dagger} \mathbf{R}_{\mathbf{x x}}^{k} \overline{\mathbf{H}}_{k} \mathbf{W}_{k} \mathbf{E}_{k}\right)
$$

We assume that the signal intended for the $k$ th MS is a Gaussian signal. As a result, (20) can be simplified to

$$
\begin{aligned}
C_{k} & =\log _{2} \operatorname{det}\left(\mathbf{I}+\frac{p_{k}}{\sigma_{n}^{2}} \mathbf{W}_{k}^{\dagger} \overline{\mathbf{H}}_{k}^{\dagger} \overline{\mathbf{H}}_{k} \mathbf{W}_{k}\right) \\
& =\log _{2} \operatorname{det}\left(\mathbf{I}+\frac{p_{k}}{\sigma_{n}^{2}} \mathbf{W}_{k}^{\dagger} \mathbf{C}_{B S}^{\dagger} \mathbf{H}_{k}^{\dagger} \mathbf{C}_{M S}^{\dagger} \mathbf{C}_{M S} \mathbf{H}_{k} \mathbf{C}_{B S} \mathbf{W}_{k}\right)
\end{aligned}
$$

For high SNRs, (21) can be further simplified and given by

$$
\begin{aligned}
C_{k} \approx & \log _{2} \operatorname{det}\left(\frac{p_{k}}{\sigma_{n}^{2}} \mathbf{W}_{k}^{\dagger} \mathbf{C}_{B S}^{\dagger} \mathbf{H}_{k}^{\dagger} \mathbf{C}_{M S}^{\dagger} \mathbf{C}_{M S} \mathbf{H}_{k} \mathbf{C}_{B S} \mathbf{W}_{k}\right) \\
= & \log _{2} \operatorname{det}\left(\frac{p_{k}}{\sigma_{n}^{2}} \mathbf{W}_{k}^{\dagger} \mathbf{H}_{k}^{\dagger} \mathbf{H}_{k} \mathbf{W}_{k}\right) \\
& \times \operatorname{det}\left(\mathbf{C}_{M S}^{\dagger} \mathbf{C}_{M S}\right) \operatorname{det}\left(\mathbf{C}_{B S}^{\dagger} \mathbf{C}_{B S}\right) \\
= & \log _{2} \operatorname{det}\left(\frac{p_{k}}{\sigma_{n}^{2}} \mathbf{W}_{k}^{\dagger} \mathbf{H}_{k}^{\dagger} \mathbf{H}_{k} \mathbf{W}_{k}\right)+\log _{2} \operatorname{det}\left(\mathbf{C}_{M S}^{\dagger} \mathbf{C}_{M S}\right) \\
& +\operatorname{det}\left(\mathbf{C}_{B S}^{\dagger} \mathbf{C}_{B S}\right)
\end{aligned}
$$

The last part of Equation (22) shows three terms contributing to the capacity. The first term represents the broadcasting capacity for the $k$ th MS without the effect of mutual coupling at BS and MS. The second and third terms represent the mutual coupling at $k$ th $\mathrm{MS}$ and BS. The effect of these terms on capacity depends on the coupling matrices at the MS and BS ends. If the product of the determinants of the mutual coupling matrices is larger than one, the effect of mutual coupling on capacity is positive. Otherwise, it is negative.

\subsection{Power Allocation Scheme}

The most straightforward power allocation scheme from $\mathrm{BS}$ to different MSs is accomplished by transmitting equal power to each MS. That is

$$
p_{k}=\operatorname{trace}\left(\mathbf{E}_{k} \mathbf{E}_{k}^{\dagger}\right)=\frac{P_{T}}{K}
$$

where $P_{T}$ is the total transmitted power at $\mathrm{BS}$ and $p_{k}$ is the power allocated to the $k$ th MS.

This power allocation scheme is simple to realize in practice. However, it does not always provide the best performance with respect to capacity. To maximize the capacity, a two-stage power allocation scheme is presented. At the first stage, the power allocation is accomplished according to the objective function at the users' level, as expressed by

$$
\begin{aligned}
& \underset{p_{1}, p_{2}, \cdots, p_{K}}{\operatorname{Max}} C_{\text {sum }}\left(p_{1}, p_{2}, \cdots, p_{K}\right) \\
& \text { Subject to } \sum_{k=1}^{K} p_{k}=P_{T}
\end{aligned}
$$

The result of (24) is the optimized power allocation for different users under service. This is the capacity-greedy power allocation scheme and is non-linear. The solution can be obtained by applying a Lagrange method.

At the second stage, the transmit power for each user can be optimized at an antenna level by using a water-filling scheme. At this stage, the power is allocated to 
different transmit antennas according to the objective function, which is described by

$$
\begin{aligned}
& p_{k}^{i}=\left(\mu_{k}-\frac{\sigma_{n}^{2}}{\lambda_{k}^{i}}\right)^{+}, i=1,2, \cdots, r \\
& \text { Subject to } \sum_{i=1}^{r} p_{k}^{i}=p_{k}
\end{aligned}
$$

where $(z)^{+}=\max (0, z)$ and $\mu_{k}$ is chosen to obey the power constraint for the $k$ th MS and $r$ is the rank of the effective channel matrix between BS and the $k$ th MS

$$
r=\operatorname{rank}\left(\mathbf{C}_{M S}^{k}\left(\mathbf{R}_{M S}^{k}\right)^{1 / 2} \mathbf{G}_{\mathbf{H}} \mathbf{R}_{B S}^{1 / 2} \mathbf{C}_{B S} \mathbf{W}_{k}\right)
$$

By applying the water-filling scheme, the capacity for $k$ th MS is

$$
C_{k}=\sum_{i=1}^{r} \log \left(1+\frac{1}{\sigma_{n}^{2}}\left(\lambda_{k}^{i} \mu_{k}-\sigma_{n}^{2}\right)^{+}\right)
$$

\section{Numerical Results}

Using the presented theory, computer simulations are performed for a multi-user MIMO system with 8 transmit antennas at BS and $3 \mathrm{MSs}$ each equipped with 2 receive antennas. It is assumed that the three MSs are scheduled and served by BS at the same time. As a result, this system is referred to as a $3 \times(2 \times 8)$ system.

Figure 1 presents the possible impact of spatial correlation and mutual coupling on the broadcasting throughput. In simulations, the dipole spacing at BS and MS is assumed to be fixed at $1.0 \lambda$ and $0.5 \lambda$, respectively.

As observed from the results presented in Figure 1, Dirty Paper Coding, where effects of spatial correlation and mutual coupling are neglected, offers the largest

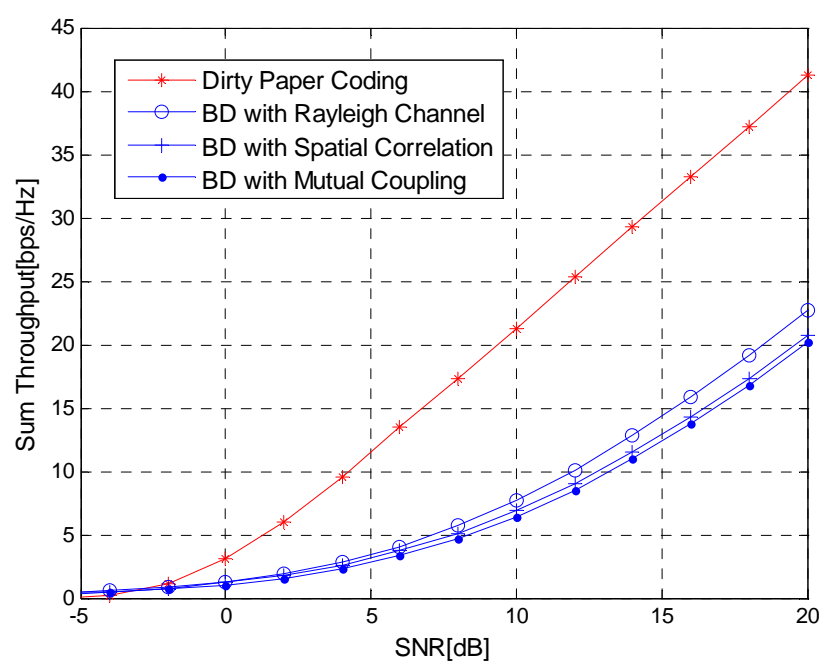

Figure 1. Broadcasting throughput for a $3 \times(2 \times 8)$ system. scheme in which spatial correlation and mutual coupling are neglected shows a reduced throughput. The throughput for BD with mutual coupling or spatial correlation included in calculations further reduces the system throughput. The differences are most pronounced at larger levels of SNR.

Figure 2 shows the effect of spatial correlation and mutual coupling on the broadcasting throughput for a $3 \times$ $(2 \times 8)$ system. The SNR is set to $10 \mathrm{~dB}$ and the unit for dipole spacing is the wavelength, represented by $\lambda$. The solid lines represent CDF of broadcasting throughput with spatial correlation only and the dotted lines are for the CDF of broadcasting throughput with spatial correlation and mutual coupling combined. It can bee seen form Figure 2 that the presence of spatial correlation and mutual coupling results in a degraded broadcasting throughput in comparison with an idealized Rayleigh channel. .

In general, spatial correlation is regarded as a negative factor in a MIMO communication system. However, mutual coupling can be seen as a positive factor at some dipole spacing. As observed in Figure 2, for the dipole spacing of $0.2 \lambda$ and $0.3 \lambda$, the existence of mutual coupling results in a higher capacity. It is interesting to note that the curve of the capacity with and without mutual coupling merge at the point of dipole spacing equal to $0.4 \lambda$. When the spacing is increased to $0.6 \lambda$, the plot representing the capacity with mutual coupling is on the left side of the curve for the capacity with correlation only. This is the case for which the presence of mutual coupling leads to a lower capacity.

Figures 3 and $\mathbf{4}$ show comparisons between capacity with spatial correlation only, and with spatial correlation plus mutual coupling, as a function of antenna element spacing.

In the presented simulation results, the SNR is set to

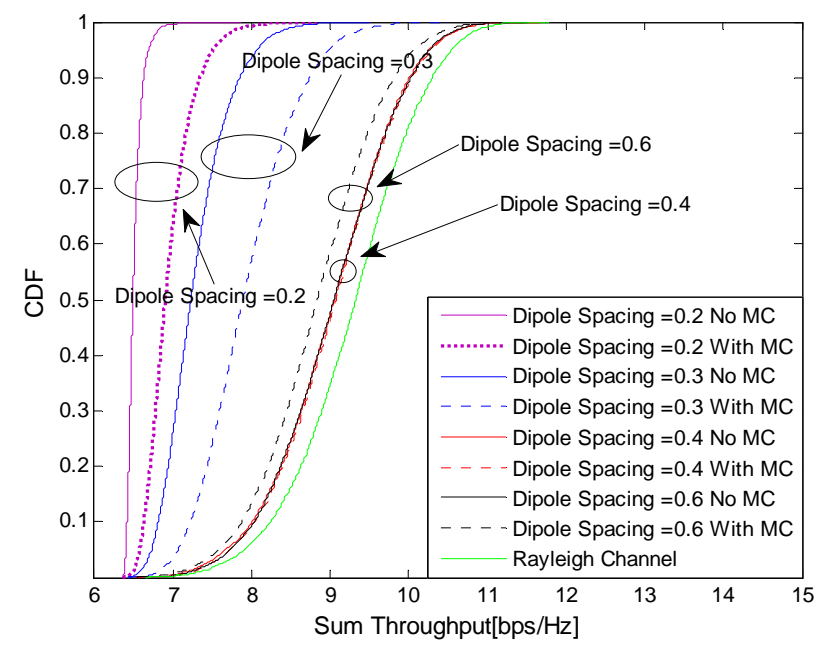

Figure 2. Broadcasting throughput CDF for a $3 \times(2 \times 8)$ system. 


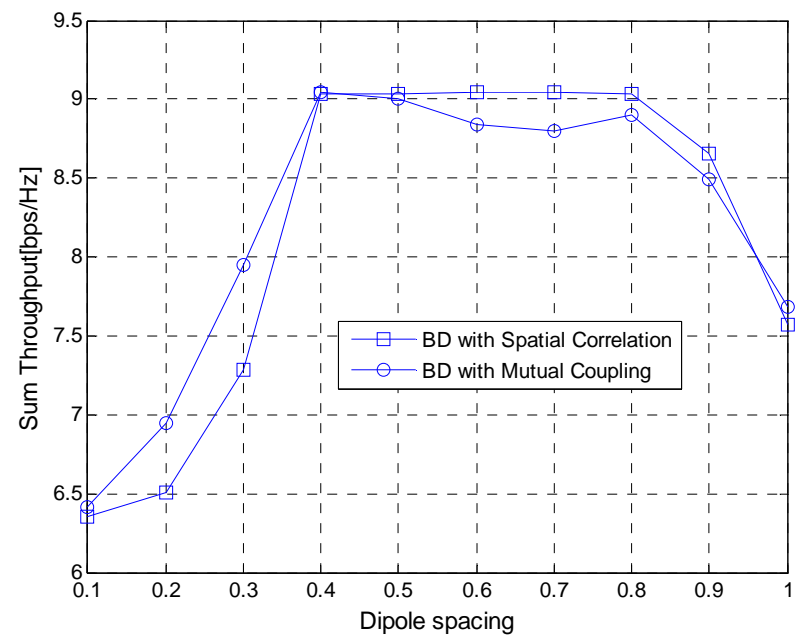

Figure 3. Broadcasting throughput vs. MS array interelement spacing for a $3 \times(2 \times 8)$ system.

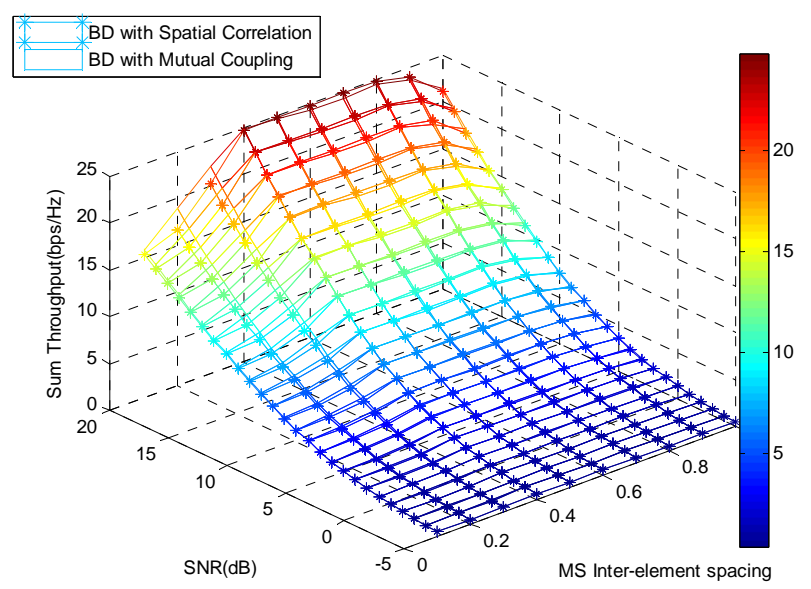

Figure 4. Broadcasting throughput vs. MS array interelement spacing and SNR for a $3 \times(2 \times 8)$ system.

$10 \mathrm{~dB}$ and the unit for dipole spacing is the wavelength, as represented by $\lambda$. The dipole spacing ranges from $0.0 \lambda$ to $1.0 \lambda$. We can see that the curves for BD with spatial correlation only and BD with spatial correlation plus mutual coupling cross at $0.4 \lambda$ and $0.95 \lambda$. For the dipole spacing range from $0.4 \lambda$ to $0.95 \lambda$, mutual coupling increases the spatial correlation level and results in a decreased capacity. In turn, when the dipole spacing ranges from $0.1 \lambda$ to $0.4 \lambda$, mutual coupling decreases the spatial correlation level and renders an increased capacity.

The results presented in Figures 5 and $\mathbf{6}$ verify the two-stage power allocation scheme described in Section 6.

One can see from results presented in Figures 5 and $\mathbf{6}$ that with or without mutual coupling, the optimized power allocation scheme leads to a higher capacity than the non-optimized one over the SNR range from $5 \mathrm{~dB}$ to $20 \mathrm{~dB}$ and the antenna spacing from $0.1 \lambda$ to $1 \lambda$. The

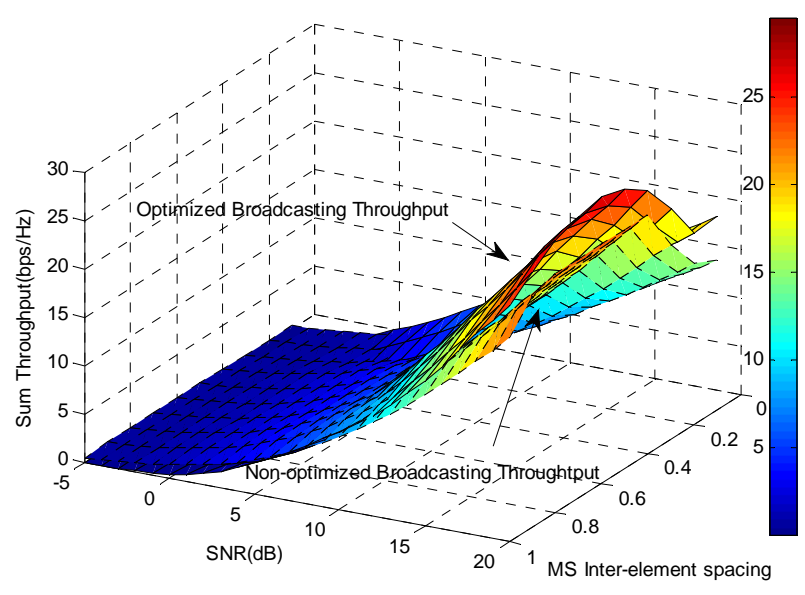

Figure 5. Comparison of optimized and non-optimized broadcasting throughput vs. MS array interelement spacing and SNR for a $3 \times(2 \times 8)$ system in the presence of spatial correlation only.

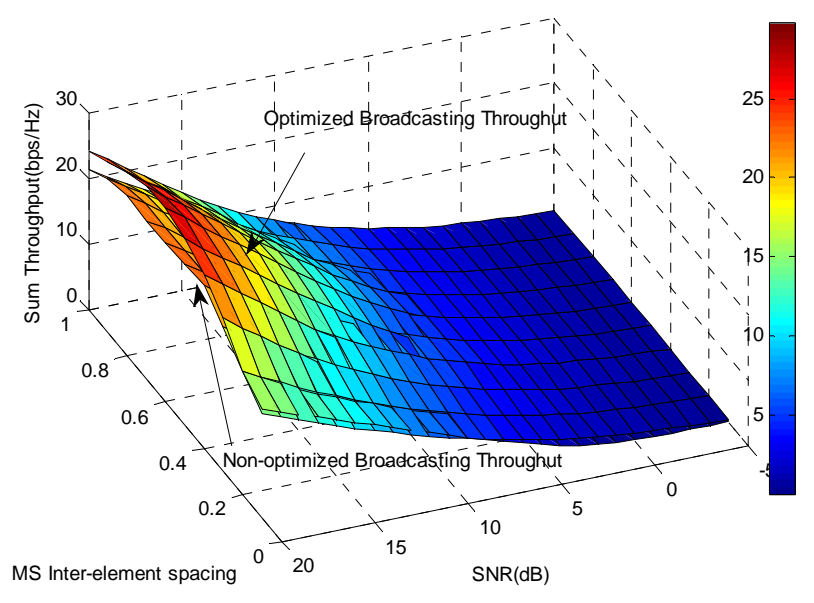

Figure 6. Comparison of optimized and non-optimized broadcasting throughput vs. MS array interelement spacing and SNR for a $3 \times(2 \times 8)$ system in the presence of spatial correlation and mutual coupling.

optimized scheme improves capacity in the presence of spatial correlation and mutual coupling. This achievement is more apparent at higher values of SNR and larger inter-element antenna spacing.

\section{Conclusions}

In this paper, investigations into the capacity of a multiuser MIMO system with block diagonalization broadcasting scheme in the presence of spatial correlation and mutual coupling have been presented. The effect of spatial correlation and mutual coupling on the broadcasting throughput for block diagonalization broadcasting has been analyzed. It has been shown by the performed computer simulations that the presence of spatial correla- 
tion leads to a decreased capacity. However, mutual coupling may have negative or positive influence of capacity. For some particular dipole spacing range, mutual coupling decreases the spatial correlation level, rendering an increased capacity. The optimized diagonalization broadcasting technique with a two-stage power allocation scheme has been proposed and verified. The presented simulations results have demonstrated a positive impact of this optimized BD scheme.

\section{Acknowledgment}

One of the authors (F. Wang) acknowledges the support of the University of Queensland in the form of International Postgraduate Research Scholarship (IPRS).

\section{References}

[1] G. J. Foschini and M. J. Gans, "On limits of wireless communications in a fading environment when using multiple antennas," Wireless Personal Communications, Vol. 6, pp. 311-335, 1998.

[2] E. Telatar, "Capacity of multi-antenna Gaussian channels," European Transactions on Telecommunications, Vol. 10, No. 6, pp. 585-596, November 1999.

[3] M. Costa, "Writing on dirty paper," IEEE Transactions on Information Theory, Vol. 49, No. 3, pp. 439-441, May 1983.

[4] W. Weingarten, Y. Steinberg, and S. Shamai, "The capacity region of the Gaussian multiple-input multiple-output broadcast channel," IEEE Transactions on Information Theory, Vol. 52, No. 9, pp. 3936-3964, September 2006.

[5] Q. H. Spencer, A. L. Swindlehurst, and M. Haardt, "Zero- forcing methods for downlink spatial multiplexing in multi-user MIMO channels," IEEE Transactions on Information Theory, Vol. 42, No. 3, pp. 461-471, February 2004.

[6] L. U. Choi and R. D. Murch, "A transmit preprocssing technique for multi-user MIMO systems using a decomposition approch," IEEE Transactions on Wireless Communications, Vol. 3, No. 1, pp. 20-24, January 2004.

[7] K. Zhang and Z. Niu, "Multiuser MIMO downlink transmission over time-varing channels," Proceedings International Conference on Communications, pp. 5514-5518, June 2007.

[8] S. Shim, J. S. Kwak, R. W. Heath, and J. Andrews, "Block diagonalization for multi-user MIMO with other-cell interference," IEEE Transactions on Wireless Communications, Vol. 7, No. 7, July 2008.

[9] S. Durrani and M. E. Bialkowski, "Effect of mutual coupling on the interference rejection capabilities of linear and circular arrays in CDMA systems," IEEE Transactions on Antennas and Propagation, Vol. 52, No. 4, pp. 1130-1134, April 2004.

[10] M. E. Bialkowski, P. Uthansakul, K. Bialkowski, and S. Durrani, "Investigating the performance of MIMO systems from an electromagenetic perspective," Microwave and Optical Technology Letters, Vol. 48, No. 7, pp. 1233 -1238 , July 2006.

[11] F. Wang, M. E. Bialkowski, and X. Liu, "Investigating the effect of mutual coupling on SVD based beamforming over MIMO channels," International Journal on Signal Processing, Vol. 3, No. 4, pp. 73-82, July 2009.

[12] C. N. Chuah, D. N. C. Tse, and J. M. Kahn, "Capacity scaling in MIMO wireless systems under correlated fading," IEEE Transactions on Information Theory, Vol. 48, pp. 637-650, March 2002. 